\title{
Additifs réducteurs de perte de charge en écoulement
}

\author{
B. Herzhaft ${ }^{1}$ \\ 1 Institut français du pétrole, 1 et 4, avenue de Bois-Préau, 92852 Rueil-M almaison - France \\ e-mail : benjamin.herzhaft@ifp.fr
}

\begin{abstract}
Résumé - Les additifs dits réducteurs de perte de charge les plus courants sont des solutions diluées de polymères, mais il existe d'autres types d'additifs, comme des particules solides (fibres) ou des tensioactifs (sous forme de micelles cylindriques). Ces additifs en concentration infinitésimale agissent en régime turbulent et peuvent diminuer les pertes de charge en écoulement jusqu'à $80 \%$ par rapport au solvant pur. Leur mécanisme d'action n'est pas clairement établi, mais c'est en interagissant avec la structure de la turbulence que ces additifs vont réduire la dissipation d'énergie et donc les pertes de charge. Entre deux régimes limites — un régime sans drag reducers et le régime asymptotique où la réduction de frottement est maximale - existe un régime intermédiaire où la réduction de frottement va dépendre des caractéristiques de l'écoulement et du type d'additif. Ce régime est caractérisé par l'apparition d'une couche intermédiaire élastique entre la couche visqueuse pariétale et le noyau turbulent de l'écoulement. Des comportements différents peuvent être observés suivant la conformation des additifs macromoléculaires. L'extension des molécules en pelotes, et/ou l'alignement des polymères étendus (ou des autres additifs anisotropes) dans l'écoulement sont caractéristiques de ce phénomène de réduction de frottement. Mots-clés : réduction de frottement, additifs, perte de charge.
\end{abstract}

Abstract - Drag Reducer Additives - Drag Reducer Additives (DRA) are mostly dilute polymeric solutions, but other kinds of additives exist, as solid particles (fibers) or tensioactive molecules (in cylindrical micelle form). These additives, present in very low concentration, act in turbulent flow regime and may decrease the flowing pressure loss by a factor up to $80 \%$ when compared to pure solvent. Their mechanism is not clearly identified but through interaction with the turbulence structure, these additives will reduce energy dissipation and thus decrease pressure losses. Between two limit regimes-a regime without drag reducer and an asymptotic regime where drag reducing is maximal-an intermediary regime exists where drag reducing will depend on flow characteristics and additive kinds. This intermediary regime is characterized by the existence of an elastic layer between the parietal viscous layer and the turbulent core. Different behaviors may be observed depending on the conformation of the macromolecular additives. Extension of coiled molecules and/or alignment of extended polymers (or other anisotropic additives) in the flow are characteristic of this drag reducing phenomenon.

Keywords: drag reducers, DRA, pressure loss. 


\section{IN TRO DUCTIO N}

Parmi les différentes solutions de réduction des pertes de charge en écoulement existantes, l'ajout d'additifs (appelés DRA, Drag Reducer Additives) en quantité infinitésimale peut être d'une grande efficacité. Des réductions de perte de charge jusqu'à $80 \%$ ont été observées.

Les additifs réducteurs de perte de charge les plus courants sont des solutions diluées de polymères qui agissent en régime turbulent. Pour une solution diluée de polymères, le coefficient de frottement est très inférieur à celui du solvant pur (quelques ppm de polymères peuvent engendrer jusqu'à $80 \%$ de réduction). Cet effet a été découvert par B.A. Toms en 1948 lors de travaux sur la dégradation mécanique des polymères, et fait toujours l'objet de nombreuses études. Cet article est une revue bibliographique des travaux existants et des mécanismes physiques proposés pour rendre compte de ce phénomène.

\section{RAPPELS}

\subsection{Coefficient de frottement}

Lors de l'écoulement d'un fluide dans une conduite cylindrique, l'écoulement est dit laminaire lorsque la trajectoire des agrégats fluides est rectiligne, confondue avec la ligne de courant correspondante. Le profil des vitesses est alors parabolique :

$$
u(r)=u_{0}\left[1-\left(\frac{r}{R}\right)^{2}\right]
$$

$r$ étant la distance à l'axe de la conduite, $R$ le rayon et $u_{0}$ la vitesse maximale :

$$
u_{0}=\frac{\Delta P D^{2}}{16 \mu L}
$$

Si l'on fait le bilan des forces extérieures s'exerçant sur le fluide, la chute de pression due aux forces de frottement peut s'évaluer à l'aide du coefficient de frottement $f$ :

$$
f=\frac{1}{4}\left(\frac{D}{L}\right) \frac{\Delta P}{\frac{1}{2} \rho u_{m}^{2}}
$$

Pour un fluide newtonien en écoulement laminaire dans une conduite cylindrique, $f$ s'exprime en fonction du nombre de Reynolds Re :

$$
\frac{f}{2}=\frac{8}{\operatorname{Re}}
$$

avec :

$$
\operatorname{Re}=\frac{\rho u_{m} D}{\mu}
$$

où $\mu$ est la viscosité du fluide, $\rho$ la densité, $u_{m}$ la vitesse moyenne sur la section de la conduite et $D$ et $L$ le diamètre et la longueur de la conduite.

Pour des nombres de Reynolds élevés, l'inertie est prépondérante et l'écoulement peut devenir turbulent : les agrégats de fluides sont alors animés d'un mouvement aléatoire (pour une conduite cylindrique, le nombre de Reynolds critique à partir duquel la turbulence apparait est généralement $\operatorname{Re}_{c}=2100$ ). Il existe au voisinage de la paroi une souscouche laminaire, puis une zone tampon, et enfin le noyau turbulent (modèle de Prandtl). L'estimation des chutes de pression en écoulement turbulent dans une conduite (et donc du coefficient $f$ ) se fait alors de façon semi-empirique (fig. 1 ). Une des relations les plus employées est due à Blasius :

$$
\frac{f}{2}=0,0395 \cdot \operatorname{Re}^{-0,25}
$$

Elle est valable jusqu'à des nombres de Reynolds de l'ordre de $10^{5}$ pour des conduites hydrauliquement lisses. Mais il existe d'autres relations semi-empiriques, plus ou moins précises ; citons Drew :

$$
\frac{f}{2}=0,0007+0,0625 \cdot \mathrm{Re}^{-0,32}
$$

et Prandtl-Karman :

$$
\frac{1}{\sqrt{f}}=4,0 \cdot \log _{10}(\operatorname{Re} \sqrt{f})-0,4
$$

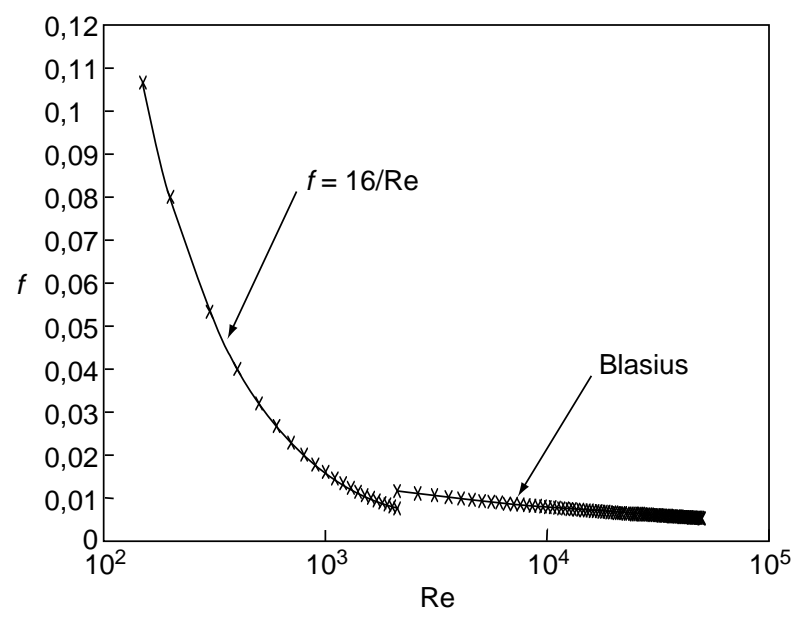

Figure 1

Variation du coefficient de frottement $f$ avec le nombre de Reynolds Re.

Variation of friction factor $f$ with Reynolds number Re. 


\subsection{Influence de la rugosité}

La rugosité de la conduite va jouer un rôle non négligeable sur les pertes de charge en écoulement turbulent. Si la hauteur moyenne des aspérités est très inférieure à l'épaisseur de la sous-couche laminaire, la rugosité n'aura pas d'influence et la conduite sera considérée comme hydrauliquement lisse. En revanche, si cette hauteur moyenne est supérieure à l'épaisseur de la sous-couche laminaire, le coefficient de friction va largement dépendre de cette rugosité moyenne. Des résultats expérimentaux obtenus par Nikuradse (1933) sur des conduites rendues artificiellement rugueuses avec une rugosité calibrée permettent de définir plusieurs domaines en fonction de la valeur du nombre de Reynolds. Pour une rugosité donnée, après une zone de transition où le coefficient de frottement $f$ augmente légèrement avec $\operatorname{Re}, f$ devient indépendant de Re pour des Re supérieurs à une valeur seuil : dans ce domaine, $f$ ne dépend plus que de la rugosité moyenne $e$ ( $f$ augmente avec $e)$ - c'est le régime hydrauliquement rugueux. Pour les conduites industrielles, il existe le diagramme de Moody représentant $f$ en fonction de Re et de $e / R$; la rugosité n'est pas uniforme et $e$ représente une rugosité équivalente.

\subsection{Profil de vitesse et couche limite}

Le profil de vitesse le long d'une paroi fait intervenir la notion de couche limite (fig. 2). En écoulement turbulent, il existe à la paroi une sous-couche laminaire où l'écoulement est bien rectiligne et où les agrégats fluides sont stables au niveau macroscopique (le mouvement aléatoire se situe au niveau des molécules avec le mouvement brownien). Audelà de cette sous-couche laminaire se situe le noyau turbulent ; les agrégats fluides sont alors animés de mouvements aléatoires, ils s'érodent au contact du fluide, s'amenuisent puis disparaissent en échangeant de la matière entre eux.

L'épaisseur de la sous-couche laminaire est très faible, la contrainte tangentielle peut être considérée comme constante et égale à $\tau_{p}$, le profil de vitesse dans la sous-couche laminaire est alors :

$$
u=\frac{\tau_{p} \cdot y}{\mu}
$$

où $y$ est la distance à la paroi et $\mu$ la viscosité. La continuité de la contrainte tangentielle à la paroi fait qu'il est possible de définir une vitesse fictive de fluctuation turbulente $u^{*}$ au contact de la sous-couche laminaire :

$$
u^{*}=\sqrt{\frac{\tau_{p}}{\rho}}
$$

que l'on appelle vitesse de frottement.

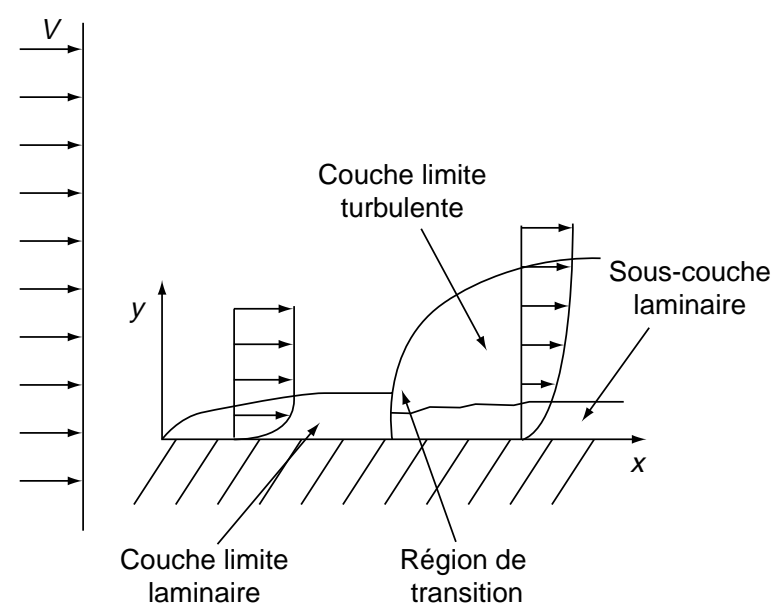

Figure 2

Théorie de la couche limite.

Boundary layer theory.

Les vitesses peuvent alors être adimensionnées par $u^{*}$ et les longueurs par:

$$
l^{*}=\frac{\mu}{\rho \cdot u^{*}}
$$

appelée longueur de friction (remarquons que $u^{*}$ peut s'exprimer en fonction de la vitesse moyenne et du coefficient de frottement ) :

$$
u^{*}=u_{m} \sqrt{\frac{f}{2}}
$$

Il est possible alors d'exprimer le profil de vitesse dans la sous-couche laminaire sous une forme adimensionnelle :

$$
\frac{u}{u^{*}}=\frac{y \cdot u^{*}}{\frac{\mu}{\rho}}
$$

Dans le noyau turbulent, le modèle de Prandtl est utilisé pour déterminer le profil de vitesse. Prandtl introduit la notion de parcours de mélange (distance que peut parcourir un agrégat fluide avant de s'éroder). Le flux de quantité de mouvement perpendiculairement à $y$ est équivalent à une contrainte instantanée. En explicitant cette contrainte, il est possible d'obtenir une relation nous donnant le profil de vitesse dans le noyau turbulent :

$$
\frac{u}{u^{*}}=2,5 \cdot \ln \frac{u^{*} \cdot y}{\frac{\mu}{\rho}}+5,5
$$


Une zone de transition existe entre la sous-couche laminaire et le noyau turbulent, et Prandtl propose la formule empirique de raccordement :

$$
\frac{u}{u^{*}}=5 \cdot \ln \frac{u^{*} \cdot y}{\frac{\mu}{\rho}}-3,05
$$

Notons que d'autres profils de vitesse sont proposés par différents auteurs.

\section{ADDITIFS DRAG REDUCERS}

Selon l'effet découvert par Toms en 1948, les solutions diluées de polymères vont présenter des coefficients de frottement $f$ bien inférieurs aux coefficients de frottement du solvant pur, alors même que les quantités de polymères mises en jeu sont trop faibles pour faire varier significativement les propriétés du fluide. Cette réduction de $f$ en régime turbulent va exister jusqu'à des Re critiques où $f$ augmentera, ces $\operatorname{Re}$ critiques correspondant à une dégradation du polymère. Cet effet de réduction de frottement peut également être obtenu avec des additifs solides, comme des suspensions de fibres. Le mécanisme responsable de cette diminution de frottement en écoulement turbulent n'est pas clairement défini.

\subsection{Drag reduction par une solution diluée de polymères}

Depuis 1948, date à laquelle Toms a mis en évidence cet effet de réduction de frottement, de nombreux travaux ont été consacrés à l'étude de ce phénomène. Pourtant, il n'existe pas à l'heure actuelle de modèle universellement accepté pour expliquer ce mécanisme. Une interaction entre le polymère et la structure de la turbulence semble être à l'origine du phénomène. Un mécanisme «complet» devrait faire intervenir l'influence de la structure et la composition du polymère, la microstructure, les interactions polymèressolvant, la rigidité ou la flexibilité de la macromolécule, etc.

Les polymères utilisés peuvent être de plusieurs types : des polyélectrolytes anioniques et cationiques, des polyampholytes, à des concentrations de l'ordre de quelques ppm, ou encore, plus récemment, des polyacrylamides modifiés hydrophobes. Il est possible de déterminer une courbe universelle de réduction de frottement en traçant \% DR (pourcentage de drag reduction par rapport à l'écoulement sans additifs) en fonction de $[\eta] C$ (viscosité intrinsèque multipliée par la concentration).

Initialement développés pour les milieux aqueux (par exemple pour les lances à incendie), les additifs drag reducers existent également en milieu organique (principalement sous forme de polyoléfines) et sont utilisés dans les applications pétrolières pour réduire les pertes de charge dans les pipelines. Les premiers développements dans ce domaine sont dus à Conoco qui a utilisé les drag reducers pour le pipeline Trans-Alaska (additif fourni par Baker) dans les années 70 et les a développés pour des applications offshore dans les années 80 (Beaty et al., 1984).

\subsubsection{O bservations phénoménologiques}

Les additifs réducteurs de frottement agissent en modifiant le comportement à la paroi. Cela a été démontré par Wells et Spangler (1967) par injection de petites quantités de solution de polymères à différents endroits de la conduite : une injection à la paroi entraîne une réduction de frottement immédiate, alors qu'avec une injection au centre de la conduite, la réduction de frottement correspondante est retardée, le temps que la solution de polymères diffuse jusqu'à la paroi. Virk (1975) a étudié en détail ce phénomène de drag reduction et a en particulier caractérisé expérimentalement le profil de vitesse dans la région proche de la paroi. Il a mis en évidence la présence d'une sous-couche laminaire et d'un noyau turbulent classiques, et l'existence d'une couche intermédiaire élastique caractéristique du phénomène. La vitesse moyenne du fluide dans cette couche élastique est supérieure à la vitesse du fluide dans la région newtonienne turbulente. Des mesures de profils de vitesse effectuées par Virk donnent pour cette sous-couche viscoélastique (au maximum de réduction de frottement) :

$$
\frac{u}{u^{*}}=11,7 \cdot \ln \frac{u^{*} \cdot y}{\frac{\mu}{\rho}}-17
$$

D'après le modèle de Virk, cette zone élastique est créée par la stimulation des molécules de polymères par l'écoulement turbulent ; sa taille augmente avec la réduction de frottement, croissant jusqu'à occuper toute la section de la conduite pour le maximum de réduction de frottement. Virk observe que la réduction de frottement est perceptible à partir d'une contrainte de cisaillement à la paroi minimale $\tau_{w}$, cette « contrainte seuil » (Onset Wall Shear Stress) ne dépendant pas de la taille de la conduite pour un polymère donné. Par exemple, pour des solutions de polyéthylène oxyde dans de l'eau et du benzène, Virk donne la corrélation suivante :

$$
\tau_{w}=4,4 \cdot \frac{10^{6}}{R_{G}^{3}}
$$

où $R_{G}$ est le rayon de giration du polymère.

Une autre observation phénoménologique d'importance est l'existence d'un maximum de réduction de frottement (Maximum Drag Reduction Asymptote, MDRA) que Virk caractérise par un coefficient de frottement $f_{m}$ tel que :

$$
\frac{1}{\sqrt{f_{m}}}=19,0 \cdot \log _{10}(\mathrm{Re}) \sqrt{f_{m}}-32,4
$$

La MDRA donne le coefficient de frottement minimum que l'on peut atteindre à un nombre de Reynolds donné. 
Il existe donc trois régimes : un premier régime où il n’y a pas de drag reduction, un deuxième régime de drag reduction où le coefficient de frottement dépend de nombreuses variables (caractéristiques du polymère, diamètre du tube et intensité de la turbulence), et enfin un troisième régime asymptotique où l'on observe un maximum de réduction de frottement : la MDRA. Le régime de drag reduction, appelé régime polymérique, peut être visualisé dans un diagramme utilisant les coordonnées de Prandtl-Karman $(1 / \sqrt{f}$ en fonction de $: \operatorname{Re} \sqrt{f}):$ il se situe entre la droite représentant la loi de Prandtl-Karman (régime sans drag reduction) et la courbe caractéristique de la MDRA (fig. 3-5).

\subsubsection{Mécanismes physiques proposés}

Le phénomène de turbulence, longtemps décrit comme chaotique et nécessitant un traitement stochastique, possède en fait une certaine structure organisée : l'éjection régulière de «bouffées » de fluide propulsées de la couche près de la paroi vers le cœur de l'écoulement est caractéristique de cette organisation, ce phénomène possédant une fréquence temporelle et spatiale. L'interaction entre ces paquets de fluides et l'écoulement principal est souvent proposée comme étant le moteur de la turbulence.

Achia et Thompson (1977) ont étudié la structure de la turbulence pour des écoulements de solutions d'additifs réducteurs de frottement. Ils ont utilisé une technique de visualisation par interférométrie. Leurs résultats montrent que les drag reducers modifient fortement la structure de l'écoulement près de la paroi, et en particulier diminuent la

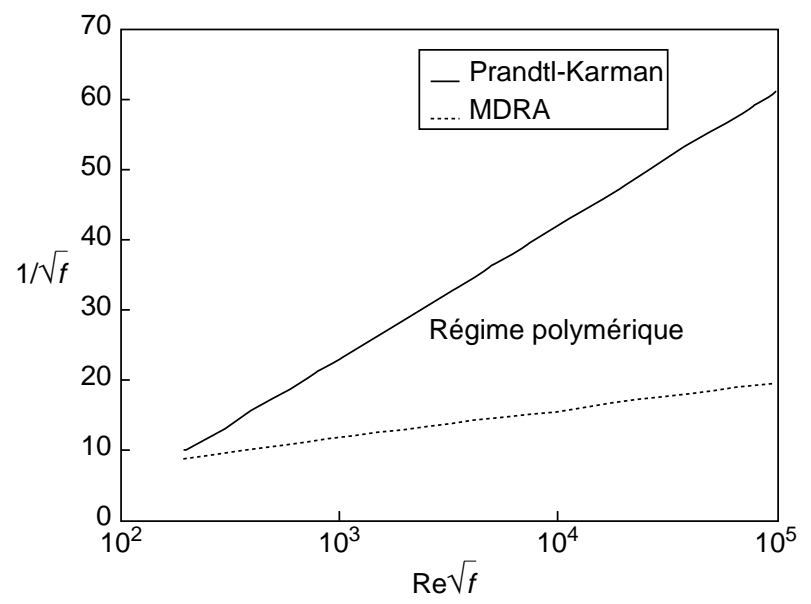

Figure 3

Réduction de frottement, régime polymérique, coordonnées de Prandtl-Karman.

Drag reducing, polymeric regime, Prandtl-Karman coordinates.

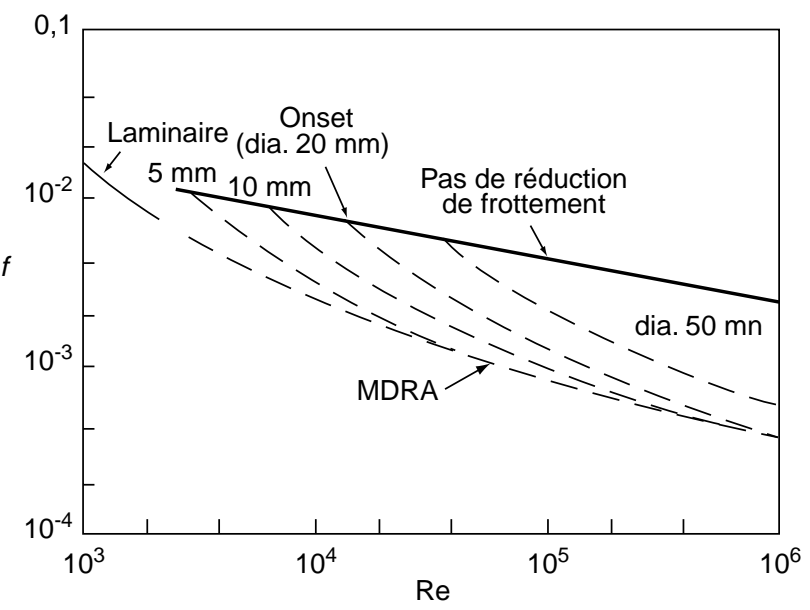

Figure 4

Variation du coefficient de frottement d'une solution de polymères drag reducer, influence du diamètre de la conduite et MDRA. D'après Tanner (1988).

Variation of a DRA polymer solution friction factor, influence of pipe diameter and MDRA. From Tanner (1988).

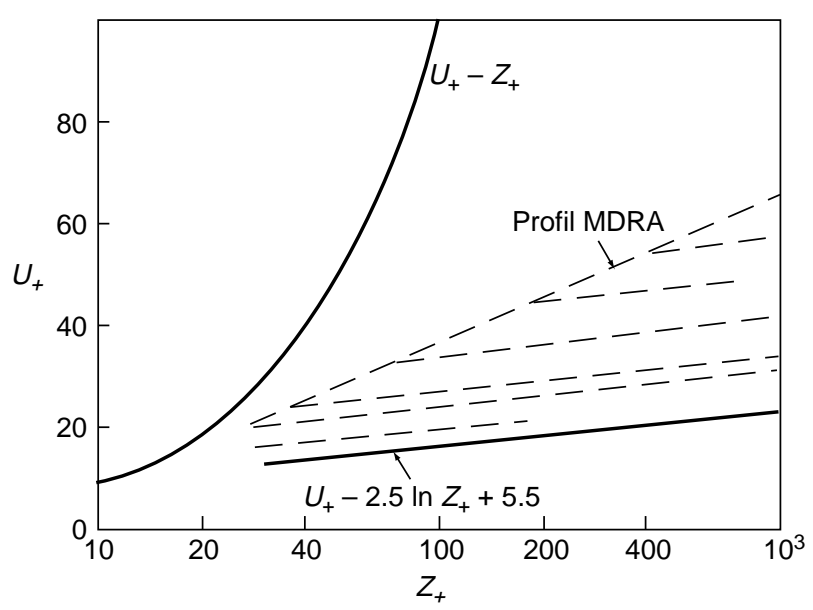

Figure 5

Profils de vitesse correspondant aux résultats de la figure 4 . D'après Tanner (1988).

Velocity profiles corresponding to results of Figure 4. From Tanner (1988).

fréquence d'éjection des «paquets » de fluides et augmentent leur espacement dans l'écoulement. La couche pariétale serait donc stabilisée par les additifs drag reducers qui réduisent l'intensité de la turbulence (si celle-ci est définie par les fluctuations de vitesse), ou plutôt réduisent la dissipation d'énergie par turbulence.

Cependant, cette explication est controversée : Virk (1975) montre que la réduction provient plus d'un «découplage » entre les composantes axiales et radiales de la turbulence que d'une diminution d'intensité (le coefficient de corrélation 
entre les deux vitesses étant fortement réduit par rapport à l'écoulement sans drag reducers).

La réduction de frottement provient donc de l'interaction entre les macromolécules et la turbulence dans la couche «élastique » voisine de la couche pariétale. Cette couche est située à une certaine distance de la paroi et débute à $y^{+}=15\left(y^{+}=y u^{*} / v\right.$ étant la coordonnée réduite) (Virk, 1975).

De nombreux auteurs ont relevé la valeur très élevée de la viscosité élongationnelle de ces solutions diluées de polymères (Baid et Metzner, 1977 ; James et Saringer, 1980). Les macromolécules peuvent alors s'allonger dans l'écoulement élongationnel et interagir avec l'écoulement turbulent, provoquant la réduction de traînée.

D'autres hypothèses attribuent à la haute résistance du polymère à l'extension l'inhibition des vortex caractéristiques de la turbulence. Cependant, le rapport des tailles caractéristiques des macromolécules (rayons de giration) et des tailles caractéristiques de tourbillons est de $10^{-2}$, les molécules isolées sont donc trop petites pour interagir avec les tourbillons. L'existence d'agrégats ayant un volume hydrodynamique important pourrait empêcher la formation de tourbillons à petite échelle et réduire ainsi l'intensité de la turbulence : la réduction de trainée proviendrait alors de l'interaction entre ces agrégats et la structure hydrodynamique.

D'autres mécanismes plus controversés ont été proposés, tels que le piégeage des macromolécules à la paroi en fonction des conditions de rugosité, la surface résultante (paroi + polymères) étant alors plus lisse et diminuant la traînée.

Quoi qu'il en soit, il est possible de dégager quelques caractéristiques générales du mécanisme de drag reduction. D'abord, cet effet provient d'une interaction entre les macromolécules et la structure de la turbulence au voisinage de la paroi. Cependant, cette interaction n'existe que dans la souscouche «élastique » adjacente à la sous-couche laminaire pariétale. Cette sous-couche élastique, caractéristique du phénomène de drag reduction, prend naissance au voisinage de $y^{+}=15$ ( $y^{+}$étant l'ordonnée adimensionnée), où la production et la dissipation d'énergie turbulente sont à leur maximum.

\subsection{Drag reduction par d'autres additifs}

\subsubsection{Additifs solides}

Le phénomène de drag reduction a également été observé avec d'autres types d'additifs, comme des particules solides anisotropes. Les premiers travaux à ce sujet datent de 1906 (Blatch) et 1931 (Forrest et Grierson) et concernent des suspensions de fibres. Les additifs solides présentent un avantage certain sur les additifs polymériques: ils sont plus solides, moins susceptibles de se dégrader avec l'écoulement et ils peuvent être séparés du fluide plus facilement. En contrepartie, des quantités plus importantes d'additifs (par rapport aux additifs polymériques) sont nécessaires pour un niveau donné de réduction de frottement, mais cette différence tend à diminuer lorsque la taille de l'écoulement augmente. L'origine de cet effet reste également contestée, mais semble provenir, comme pour les additifs macromoléculaires, de phénomènes à la paroi (Kale et Metzner, 1976) et de l'alignement des fibres dans l'écoulement. Certains auteurs ont mis en évidence un effet accru lorsque les additifs fibreux sont employés simultanément à des additifs macromoléculaires (Kale et Metzner, 1974).

\subsubsection{Additifs tensioactifs}

La réduction de perte de charge en écoulement lorsqu'on ajoute une solution de surfactants est un phénomène connu depuis longtemps (Mysels, 1949). Il existe pourtant peu d'études concernant ces additifs par rapport à la littérature existante sur les additifs macromoléculaires. Un des avantages de ce type d'additifs est leur «durée de vie » : comparativement aux solutions de polymères, ils présentent une grande résistance aux dégradations mécaniques et ont donc une « durée de vie » plus grande (Bewersdorff, 1988).

Une molécule de surfactant ou tensioactif est constituée d'une chaîne à caractère hydrophobe et d'un groupement polaire fortement hydrophile. De par leur structure chimique, ces molécules vont avoir tendance à se placer à une interface. Au-dessus d'une certaine concentration appelée $C M C$ (concentration micellaire critique), les molécules de tensioactifs ont tendance à former des structures appelées micelles avec les parties polaires tournées vers le solvant de façon à minimiser la surface offerte par les parties hydrophobes au solvant. Suivant la température, la nature et la concentration des molécules de tensioactif, la taille et la forme des micelles formées vont varier (fig. 6).

Les solutions de surfactants efficaces en drag reduction sont caractérisées par la présence de micelles cylindriques. Elles ne sont donc efficaces que dans une certaine plage de températures et une certaine concentration en surfactants. Les concentrations typiques efficaces en drag reduction sont de l'ordre de 200-2000 ppm, la longueur des micelles cylindriques étant alors de l'ordre de $25-200 \mathrm{~nm}$ avec un diamètre de $2-5 \mathrm{~nm}$ (Bewersdorff, 1990). L'alignement progressif des micelles cylindriques dans la direction de l'écoulement semble corrélé avec l'effet de drag reduction. Au-dessus d'un certain cisaillement critique, l'effet de drag reduction diminue et l'on observe une forte augmentation de la viscosité de la solution, ce qui est expliqué par une destruction de l'alignement des micelles dans l'écoulement.

\subsection{Ca ractéristiques communes}

Virk fait une distinction entre deux types de drag reduction existant dans le régime polymérique, le type $\mathrm{A}$ et le type $\mathrm{B}$ (Virk, 1990). Le type A est caractéristique d'additifs macromoléculaires en pelotes statistiques, et se traduit dans un diagramme de Prandtl-Karman par des courbes de drag 


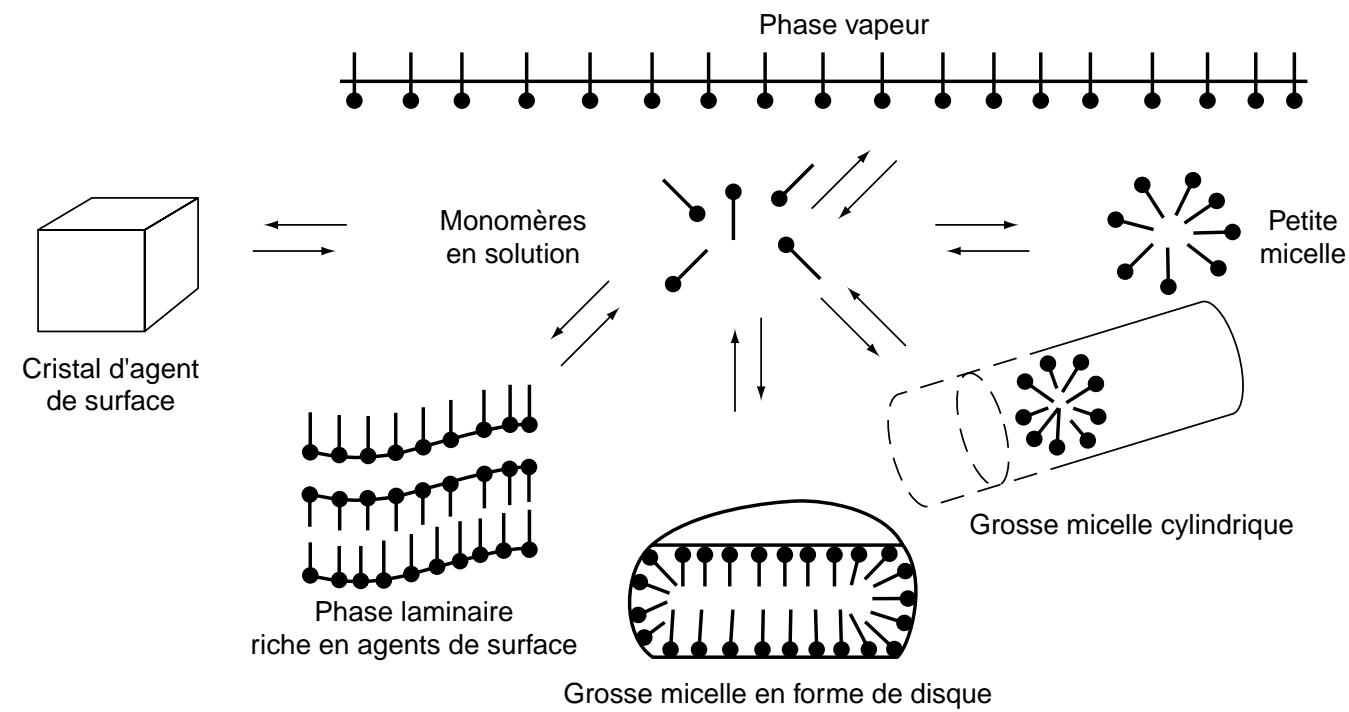

Figure 6

Représentation schématique des diverses structures auxquelles participent les agents tensioactifs en solution aqueuse. D'après Briant (1988). Schematic representation of various structures characteristic of tensioactive agents in solution. From Briant (1988).

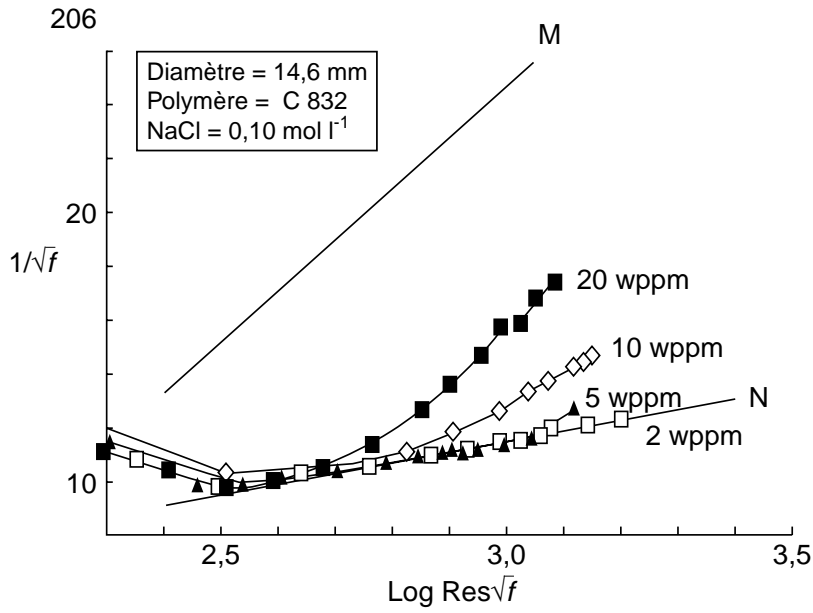

Figure 7

Polymères en pelotes statistiques, diagramme de PrandtlKarman. D'après Virk (1990).

Polymers in statistic coils, Prandtl-Karman diagram. From Virk (1990).

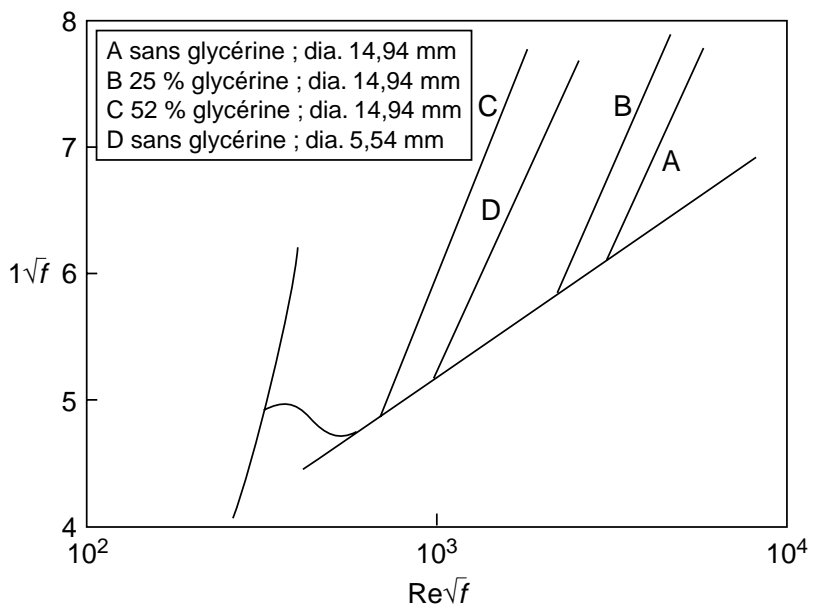

Figure 8

Polymères en pelotes statistiques : onset de la réduction de traînée. D'après Berman (1978).

Polymers in statistic coils: onset of drag reduction. From Berman (1978). reduction partant d'un même point sur la droite de PrandtlKarman (représentant le régime sans drag reducer) et dont les pentes augmentent avec la concentration (fig. 7, $\mathrm{N}$ la loi de Prandtl-Karman et M la MDRA). Pour ce type de molécules, il existe donc un «point de départ» (onset) pour la réduction de traînée dépendant du type de macromolécule utilisé et des caractéristiques de l'écoulement (fig. 8). Le type $\mathrm{B}$ se rencontre avec des polymères en extension, mais également avec des additifs différents, comme des fibres solides ou des micelles de tensioactifs. Il se traduit dans un diagramme de Prandtl-Karman par des courbes de drag reduction à peu près parallèles à la courbe de PrandtlKarman, et déplacées vers le haut lorsque la concentration augmente (fig. 9).

Pour le type $\mathrm{B}$, à un niveau de turbulence donné (à une valeur de $\operatorname{Re} \sqrt{f}$ donnée), le niveau de réduction de frottement augmente pratiquement linéairement avec la concentration en additifs. 


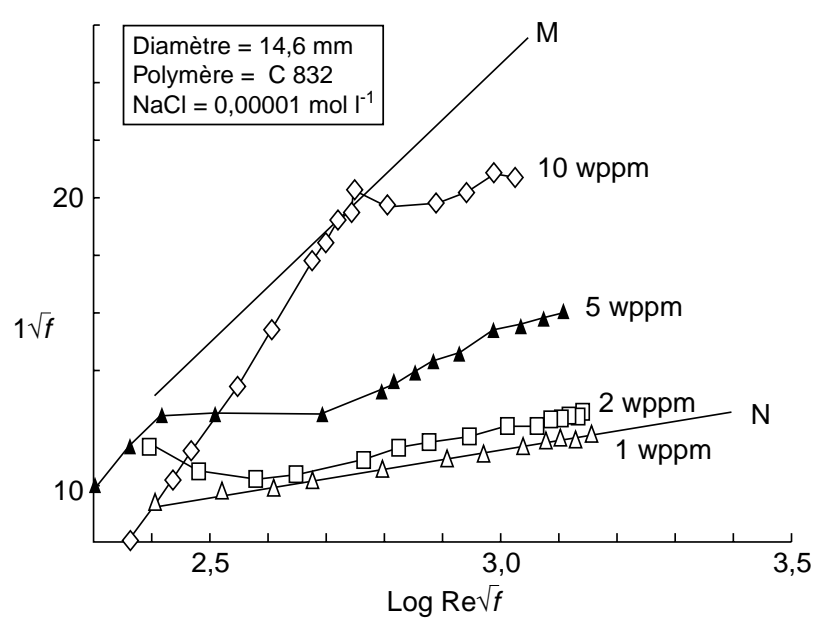

Figure 9

Polymères en configuration étendue, diagramme de PrandtlKarman. D'après Virk (1990).

Polymers in extended conformation, Prandtl-Karman diagram. From Virk (1990).

\section{Onset et limites}

Pour des macromolécules en pelotes sphériques, il n'y a pas de drag reduction tant qu'elles n'interagissent pas avec l'écoulement. À un certain niveau de turbulence, les macromolécules commencent à interagir avec l'écoulement (onset). Cette interaction peut se traduire par une extension de la molécule dans l'écoulement élongationnel, ou l'agrégation de plusieurs molécules de façon à former un agrégat de taille similaire à celle des tourbillons turbulents. Berman et George (1974) ont montré qu'il y avait une corrélation entre l'échelle de temps des molécules individuelles et l'échelle de temps de l'écoulement (fig. 7). Or, les solutions de macromolécules présentent généralement une polydispersité en taille et donc plusieurs échelles de temps. Hunston et Reischman (1975) ont montré que le phénomène de drag reduction était régi par les fractions de macromolécules de plus haut poids moléculaire.

Que ce soit pour des macromolécules en pelotes statistiques (type A) ou étendues dans l'écoulement (type B), l'intensité de la réduction de traînée augmente avec la concentration et le poids moléculaire jusqu'à atteindre une limite.

À nombre de Reynolds élevé, on observe dans les deux cas une diminution du niveau de drag reduction. Pour les macromolécules en conformation étendue (comme les polyélectrolytes), le comportement est similaire à celui des fibres solides ; l'une des explications avancées est qu'à nombre de Reynolds très élevé, les objets anisotropes (fibres, micelles de tensioactifs ou polymères étendus) perdent leur orientation préférentielle dans l'écoulement. Pour les macromolécules en pelotes statistiques, la diminution de l'intensité de drag reduction à Re élevé semble provenir d'une dégradation des molécules due à l'écoulement (Berman, 1978).

\subsection{Drag reduction hétérogène}

Alors que le phénomène de drag reduction classique concerne des solutions extrêmement diluées de polymères, des études récentes ont montré que l'injection d'une solution hautement concentrée de polymères au cœur d'un écoulement turbulent pouvait réduire les pertes de charge en écoulement d'un facteur important.

La première mise en évidence de ce phénomène est due à Vleggaar et Tels (1973). La concentration de la solution de polymères injectée au centre de l'écoulement est de 0,5 à $1 \%$. Cette solution forme un filament qui conserve son intégrité jusqu'à des distances très éloignées du point d'injection : nous sommes donc en présence d'un système hétérogène donnant lieu à des réductions de perte de charge importantes.

Le mécanisme d'action reste controversé. D'après leurs observations, Vleggaar et Tels (1973) ont conclu que, la solution de polymères étant concentrée dans un filament au centre de l'écoulement, la réduction de traînée devait provenir de l'interaction de ce filament viscoélastique avec la turbulence du cœur de l'écoulement, hypothèse confirmée par Bewersdorff (1982) et Usui (1990). Selon une hypothèse différente, de petites quantités de polymères diffuseraient du filament central vers la région de l'écoulement proche de la paroi, ce qui suffirait à provoquer la réduction de frottement ; le mécanisme serait alors similaire au mécanisme de drag reduction classique. McComb et Rabie (1982) proposent cette hypothèse à partir de mesures de profils de concentration, et plus récemment, Smith et Tiederman (1991) l'ont confirmée à partir d'expériences de diffusion de traceur. Hoyt et Sellin (1991) attribuent le phénomène de drag reduction à la diffusion de molécules de polymères depuis le filament central vers la région de l'écoulement proche de la paroi pour des nombres de Reynolds élevés, alors qu'à de faibles nombres de Reynolds, cet effet serait dû à l'interaction du filament central avec la turbulence à grande échelle du cœur de l'écoulement.

\section{CONCLUSION}

Cette revue sommaire montre que, bien que connu depuis une cinquantaine d'années, le phénomène de drag reduction reste un sujet de recherche vivant dont le mécanisme n'est pas clairement élucidé.

Plusieurs types d'additifs donnent lieu à cette réduction de frottement en régime turbulent : les additifs macromoléculaires, que ce soit des polymères en pelotes statistiques ou des polymères en conformation étendue, les tensioactifs formant des micelles cylindriques, les additifs solides, comme des fibres. Nous avons vu également qu'il existait un phénomène de drag reduction hétérogène avec injection d'une solution concentrée de polymères au cœur de l'écoulement. 
Quelques caractéristiques du mécanisme par lequel ces additifs, en quantités infinitésimales, donnent lieu à d'importantes réductions de perte de charge sont acceptées par la grande majorité des auteurs.

Entre deux régimes asymptotiques (le régime sans drag reducers et le régime de réduction de frottement maximum) existe un régime intermédiaire appelé régime polymérique où l'intensité de la réduction de frottement va dépendre du type d'additifs, de leur concentration et des caractéristiques de l'écoulement. Nous avons vu qu'il existe deux types différents de comportement (le type A et le type B) dans ce régime polymérique suivant le type d'additifs.

Concernant le mécanisme, l'effet de drag reduction provient d'une interaction entre les additifs et la structure de la turbulence au voisinage de la paroi. Au «départ» (onset) de la réduction de frottement (pour le type A), il existe une corrélation entre la taille caractéristique des macromolécules et l'échelle de longueur de la turbulence. L'étude des profils de vitesse montre que, entre la couche visqueuse pariétale et le noyau turbulent, apparaît une sous-couche élastique caractéristique du phénomène, l'interaction entre les additifs et l'écoulement se situant principalement dans cette zone tampon où la production et la dissipation d'énergie associées à la turbulence sont à leur maximum. La structure de la turbulence est modifiée par ces interactions ; en particulier, l'anisotropie de l'écoulement turbulent est fortement augmentée.

L'analyse du type B de drag reduction montre que ce régime est caractérisé par l'alignement des additifs anisotropes dans l'écoulement élongationnel, l'espacement entre les particules étant inférieur à leur longueur.

Pour de nombreux auteurs, le phénomène de drag reduction est principalement dû à l'extension des macromolécules dans l'écoulement et à l'alignement des additifs (voir discussion dans Gyr, 1990) ; le type A et le type B définis par Virk ne seraient alors que deux aspects du même mécanisme. La drag reduction semble dans tous les cas être fortement liée à la présence d'objets présentant une forte anisotropie (fibres, polymère étendus, etc.).

\section{REMERCIEMEN TS}

L'auteur tient à remercier M. Moan, professeur à l'Université de Bretagne occidentale, pour son aide.

\section{RÉFÉREN CES}

Achia, B.U. et Thompson, D.W. (1977) Structures of Turbulent Boundary in Drag-Reducing Pipe Flow. J. Fluid Mech., 81, 439464.

Baid, K.M. et Metzner, A.B. (1977) Rheological Properties of Dilute Polymer Solutions Determined in Extensional and in Shearing Experiments. Trans. Soc. Rheol., 21, 237-260.
Beaty, W.R., Johnston, R.L., Kramer, R.L., Warnock, L.G. et Wheeler, G.R. (1984) Drag Reducers Increase Flow in Offshore Pipelines without Additional Expansion. Oil \& Gas Journal, 71-74.

Berman, N.S. et Georges, W.K. (1974) Onset of Drag Reduction in Dilute Polymer Solutions. Phys. Fluids, 17, 250-251.

Berman, N.S. (1978) Drag Reduction by Polymers. Ann. Rev. Fluid Mech., 10, 47-64.

Bewersdorff, H.W. (1982) Rheol. Acta, 21, 587-589.

Bewersdorff, H.W. (1990) Structure of Turbulence and Drag Reduction, IUTAM Symposium, Zurich, Gyr, A. (éd.), 266, 941.

Bewersdorff, H.W. et Ohlendorf, D. (1988) Colloid and Polymer Sci., 266, 941.

Blasius, H. (1913) Mitt. Forschungarb., 131, 1.

Blatch, N.S. (1906) Water Filtration at Washington. Trans. ASCE, 57, 400.

Briant, J. (1988) Phénomènes d'interface, agents de surface, Éd. Technip.

Forrest, F. et Grierson, G.A.H. (1931) Friction Losses in Cast Iron Pipes Carrying Paper Stock. Paper Trade J., 298.

Gyr, A. (1990) Structure of Turbulence and Drag Reduction, IUTAM Symposium, Zurich, Gyr, A. (éd.).

Hoyt, J.W. et Sellin, R.H.J. (1991) Rheol. Acta, 30, 307.

Hunston, D.L. et Reischman, M.N. (1975) The Role of Polydispersity in the Mechanism of Drag Reduction. Phys. Fluids, 18, 1626-1630.

James, D.F. et Saringer, J.H. (1980) Extensional Flow of Dilute Polymer Solutions. J. Fluid Mech., 97, 655-671.

Kale, D.D. et Metzner, A.B. (1974) Turbulent Drag Reduction in Fiber-Polymer Systems: Specificity Considerations. AIChE J., 20, 1218.

Kale, D.D. et Metzner, A.B. (1976) Turbulent Drag Reduction in Dilute Fiber Suspensions: Mechanistic Considerations. AIChE J., 22, 4, 669-674.

McComb, W.D. et Rabie, L.H. (1982) Local Drag Reduction Due to Injection of Polymer Solutions into Turbulent Flow in a Pipe. AIChE J., 28, 547-565.

Mysels, K.J. (1949) US Patent 2492173.

Nikuradse (1933) Forschungsheft, 361, VDI Verlog, Berlin.

Smith, R.E. et Tiederman, W.G. (1991) Rheol. Acta, 30, 103-113.

Tanner (1988) Engineering Rheology, Clarendon Press, Oxford.

Toms, B.A. (1977) On the Early Experiments on Drag Reduction by Polymers. Phys. Fluids, 20, 10, II, S3.

Usui, H. (1990) Structure of Turbulence and Drag Reduction, IUTAM Symposium, Zurich, Gyr, A. (éd.).

Virk, P.S. (1975) Drag Reduction Fundamentals. AIChE J., 21, 4, 625-657.

Virk, P.S. (1990) Structure of Turbulence and Drag Reduction, IUTAM Symposium, Zurich, Gyr, A. (éd.).

Vleggaar, J. et Tels, M. (1973) Chem. Eng. Sci., 28, 965-968.

Wells, C.S. et Spangler, J.G. (1967) Injection of Drag-Reducing Fluid into Turbulent Pipe Flow of a Newtonian Fluid. Phys. Fluids, 10, 1890 .

Manuscrit définitif reçu en mai 2000 